\title{
Mitteilungen aus der Arbeitsgruppe Elektrophysiologie und Rhythmologie (AGEP)
}

Liebe Kolleginnen und Kollegen, liebe Mitglieder der AGEP!

Nach den Herztagen ist vor der Jahresstagung!

Der Herbst steht merklich vor der Tür, was man nicht nur an den Temperaturen und den deutlich kürzer werdenden Tagen merkt.

Außerdem hat uns der klinische Alltag wieder fest im Griff. Nichtsdestotrotz soll dieser Herbstnewsletter Sie auf den aktuellen Stand bringen und auf ein paar anstehende Termine hinweisen.

\section{Abstract Deadline}

Für die EHRA-Abstracts 2022 ist die Abgabe noch bis Januar 2022 möglich.

\section{EP case of the year}

In diesem Jahr plant die AGEP erstmalig den „EP case of the year" auszuloben. Zu diesem Preis wird es dann bei der Jahrestagung eine eigene Sitzung geben, in der die besten fünf Kasuistiken aus dem Bereich Elektrophysiologie vorgestellt werden. Zwei davon werden ausgewählt und prämiert. Die Deadline und alle weiteren Infos folgen demnächst. Wir freuen uns auf Ihre Einreichungen!

\section{Neuer Intensivkurs „Süd“}

Die Planungen für den kommenden EP Intensivkurs laufen auf Hochtouren, dieser wird als "Südkurs“ in München, Bad Neustadt, Wiesbaden, Heidelberg und Coburg stattfinden.

Hier schon einmal die Termine:

- 17. und 18. März 2022 (München): Organisation EP Labor

- 7. und 8. April 2022 (Heidelberg): EP Grundlagen

- 12. und 13. Mai 2022 (Coburg): Vorhofflimmern und atriale Tachykardien Coburg

- 9. und 10. Juni 2022 (Bad Neustadt):VT/VES

- 7. und 8. Juli 2022 (Wiesbaden): Klinische Elektrophysiologie

Die Anmeldung über die Seite der Akademie ist bald möglich unter https://akademie.dgk.org/ - und wie immer lohnt es sich schnell zu sein, da der Kurs traditionell immer schnell ausgebucht ist! Wir freuen uns auf Ihre Anmeldungen!

\section{Die AGEP online}

Neben den aktualisierten Inhalten auf der Homepage der AGEP können wir Ihnen auch empfehlen den Twitter handle @AGEP_DGK zu abonnieren, über den gerade während der Kongresse viele Inhalte schnell zugänglich waren! Außerdem möchten wir Ihnen die Siilo Gruppe *just beat it* empfehlen, in der spannende Befunde und EKGs in einer großen Community von >120 Elektrophysiologen diskutiert werden.

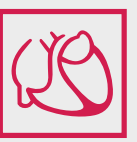

AGEP.

Arbeitsgruppe Elektrophysiologie und Rhythmologie

Deutsche Gesellschaft für Kardiologie - Herz- und Kreislaufforschung e.V.

V.i.S.d.P.

Univ.-Prof. Dr. med. Daniel Steven

Herzzentrum der Uniklinik Köln

Kerpener Str. 62, 50937 Köln

Telefon: 0221/478-32396

E-Mail:daniel.steven@uk-koeln.de

\section{Neuer Beitrag der Reihe EP Basics}

In der aktuellen Ausgabe findet sich der EP Basics-Artikel zum Thema "Leitfaden zur sicheren und effizienten Kryoballon-Vorhofflimmerablation“ - der meistgelesene Artikel der Serie „Punktionstechniken in der invasiven Elektrophysiologie" wurde "free access" gestellt: https://www.springermedizin.de/ep-basics/19564462

\section{Kooperation mit der Zeitschrift Herzschrittmachertherapie \& Elektrophysiologie (H+E)}

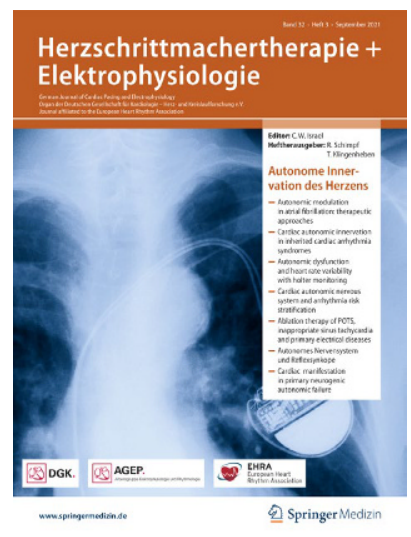

Alle DGK-Mitglieder können ein Abonnement der Zeitschrift zu vergünstigten Konditionen erwerben (54.-€ pro Jahr inkl. Mwst. und Versand). Die Abos können über die Geschäftsstelle der DGK bestellt werden (info@dgk.org).

\section{In eigener Sache}

Erzählen Sie gerne Ihren Kollegen von den Aktivitäten der AGEP und bitte vergessen Sie nicht das Pflege- und Assistenzpersonal: jeder, der an der Elektrophysiologie und Rhythmologie interessiert ist, ist in der AGEP willkommen! Mitglied zu werden und damit die AGEP bei ihren Aktivitäten zu unterstützen ist einfach: eine formlose E-Mail mit der Bitte um Aufnahme in die AG an arbeitsgruppen@dgk.org ist ausreichend.

Bleiben Sie informiert:

- www.kardiologie.org

Alle Sessions der DRT on demand verfügbar

- www.ag-ep.de

Aktuelle Informationen der AGEP

- @AGEP_DGK

AGEP auf Twitter

Bleiben Sie gesund, Ihre

Prof. Dr. Daniel Steven Sprecher der AGEP
Prof. Dr. Christian Veltmann stv. Sprecher der AGEP 